\title{
Available Bandwidth Estimation in Smart VPN Bonding Technique based on a NARX Neural Network
}

\author{
Giacomo Capizzi $^{1,2}$, Grazia Lo Sciuto ${ }^{1}$, Francesco Beritelli ${ }^{1}$, Francesco Scaglione ${ }^{1}$, Dawid Połap ${ }^{1,2}$, Kamil Książek ${ }^{2}$, \\ Marcin Woźniak ${ }^{1,2}$ \\ ${ }^{1}$ Department of Electrical, Electronics, and Informatics Engineering, \\ University of Catania, Viale A. Doria 6, 95125 Catania, ITALY \\ gcapizzi@diees.unict.it, glosciuto@dii.unict.it, francesco.beritelli@dieei.unict.it, scaglione.fnc@gmail.com \\ ${ }^{2}$ Insitute of Mathematics, Silesian University of Technology, \\ Kaszubska 23, 44-100 Gliwice, POLAND \\ dawid.polap@polsl.pl,marcin.wozniak@polsl.pl
}

\begin{abstract}
Today many applications require a high Quality of Service $(\mathrm{QoS})$ to the network, especially for real time applications like VoIP services, video/audio conferences, video surveillance, high definition video transmission, etc. Besides, there are many application scenarios for which it is essential to guarantee high QoS in high speed mobility context using an Internet Mobile access. However, internet mobile networks are not designed to support the real-time data traffic due to many factors such as resource sharing, traffic congestion, radio link, coverage, etc., which affect the Quality of Experience (QoE). In order to improve the QoS in mobility scenarios, the authors propose a new technique named "Smart VPN Bonding" which is based on aggregation of two or more internet mobile accesses and is able to provide a higher end-to-end available bandwidth due to an adaptive load balancing algorithm. In this paper, in order to dynamically establish the correct load balancing weights of the smart VPN bonder, a neural network approach to predict the main Key Performance Indicators (KPIs) values in a determinate geographical point is proposed.
\end{abstract}

Index Terms-Smart VPN Bonding, bandwidth prediction, QoS improvement, Neural Network Introduction.

\section{INTRODUCTION}

$\mathrm{N}$ OWADAYS the use of mobile Internet services, namely the use of Internet services through the data access offered by different cellular providers has experienced a significant increase. This increase is certainly due to the growing demanding needs of users to be connected anywhere and anytime, but also to the possibility of providing a connection in areas beyond reach over wired infrastructure.

There are numerous application scenarios, and others are currently under development, for the situations in which it is essential to have a stable Internet access and high performance even in the conditions of mobility:

- Wi-Fi on public transport,

- Connection between moving units and central station (e.g. Rescue units),
- Video surveillance of means of transport (e.g. Transport values),

- Telemedicine in mobility (e.g. Ambulances for first aid).

In addition, real time services like audio and video transmission (VoIP, audio/video conference, remote video surveillance, etc.) and services that require high Quality of Service (QoS) are currently having an exponential growth of usage.

A possible approach to improve network performance is based on the possibility to use a technique called VPN Bonding capable of aggregating the available Internet access (Ethernet, 3G, 4G, WiFi, etc.) with the ultimate goal to noticeably improve performance in terms of bandwidth, thus reaching ideal broadband speeds equal to the sum of the available bandwidths. In addition obviously obtaining a high fault tolerance in case of inefficiency. This is possible thanks to load balancing mechanism which acts at the level 2 capable of sorting packages on various available connections.

The basic idea involves the use of different mobile operators in order to compensate possible deficiencies of an operator, sorting the load mainly toward the available connections from other operators who at that moment and at that point offer greater performance.

Empirical studies show that in order to obtain an excellent result tending to the ideal solution, namely that of using a bandwidth equal exactly to the sum of the bandwidths offered by each access, it is essential that traffic can be balanced in a manner proportional to the performance offered by each Internet connection or improved methods [14].

Obviously, if we focus on mobility contexts in which one has to use the cellular network, it must be emphasized that the QoS (Quality of Service) offered by each access is definitely subject to greater variability when compared [21], for example, to the QoS offered by a classic ADSL. This is 
because additional factors that can affect the quality of the connection are involved in the Mobile Internet scenario: propagation conditions, interference levels, dependence on activities of other users as a shared communication channel, saturation of the cell to which it is hooked, but especially the concept of mobility, which can lead the user to move from a good coverage area to a poor radio signal coverage areas thus ensuring that the performance of the data connection may be affected.

As mentioned above, in order to cope with the variability of access conditions there should be a real-time evaluation of the QoS offered by each single data access, so as to vary in an adaptive way the weights to be assigned to the load balancing mechanism and thus try to always balance the load in an optimal manner.

It is therefore essential to identify the techniques to make an accurate QoS estimation and simultaneously have low times of convergence in highly dynamic environment.

Section II outlines the state-of-the-art bandwidth prediction techniques that use a dataset of past collected information, according to a certain point in the territory. Section III discusses in brief the Smart VPN Bonding technique [1] [2], providing an overview of the techniques so far adopted for the estimate of the QoS, in particular the available bandwidth, highlighting problems and introducing benefits that an approach through a predictive neural network could offer.

\section{RELATED WORKS}

In literature we can find different application contexts in which it is useful to perform certain actions on the basis of predictions based on the analysis of historical events. In particular, this approach is used in many contexts to ensure high QoS for network applications.

This approach, for example, can be used to improve the performance of routing algorithms, whereas the additional parameters are essential in the choice of the optimal route, such as prediction of the available bandwidth and the delay. By estimating these parameters following the values they had in the past the routing algorithm is able to select the most satisfactory path in terms of the bandwidth and the delay [4].

A similar approach could be useful to improve performance in the algorithms used in the handover mechanisms. In [5] a handover mechanism is proposed using a predictive method based on fuzzy logic, while in [6] a vertical handover algorithm is suggested based on the prediction of RSSI so as to be able to make an intelligent and flexible passage of information through different 4G wireless communication systems, reducing the switching delay in comparison with the classical algorithms of vertical handover.

In [7] and [8] the idea of building the "bandwidth map" is put forward to enhance the QoS in highly mobile environments [22]. Through the use of these maps, based on information collected in the past, one can expect the available bandwidth in a given geographical point, calculated as an average historical value, and as a consequence dynamically determine the most suitable bit rate for encoding video streaming.
In some real applications it is very useful to predict some parameters by a limited data subset. In particular some studies are concerned about the relation between packet delay and other parameters. For instance, in [9] a relation between Round Trip Time (RTT) and other geographic and network properties is investigated.

Finally, to improve prediction and reduce the error rate different approaches based on the use of Neural Networks have been studied, in particular for the purpose of the Radio Frequency (RF) power prediction [10], [11] and for the bandwidth estimation [12], [13].

\section{SMART VPN BONDING}

\section{A. Architecture}

As discussed in previous sections, the Smart VPN Bonding technique allows aggregating the resources offered by two or more mobile radio data accesses obtaining a remarkable increase in performance in terms of bandwidth. Fig. 1 indicates the proposed architecture of the Smart VPN Bonding [1], [2].

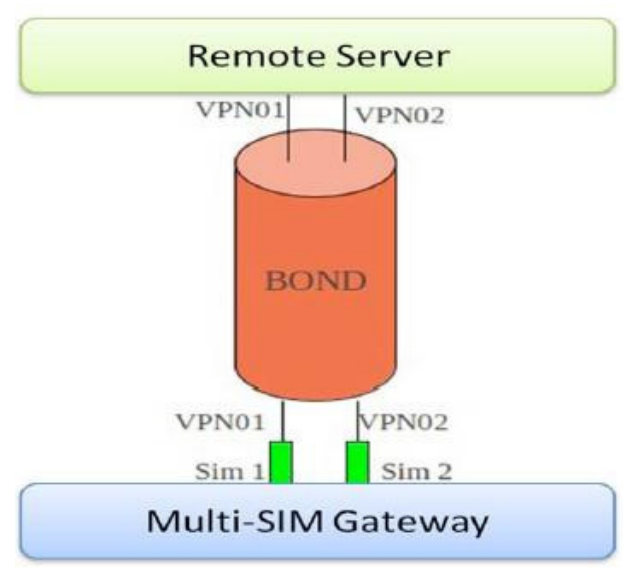

Fig. 1. Smart VPN Bonding architecture [2]

The scope is to create a VPN tunnel between each access mobile radio and the end point of the communication, i.e. the remote server. After creating the VPN tunnels they can be aggregated into a single interface with the aim to establish a broadband connection between the source and the destination. This is possible since a load balancing mechanism capable of sorting the frames into various VPN tunnels aggregated in the "Bond" interface is adopted. Obviously the performance can be boosted in terms of bandwidth if traffic is balanced in proportion to the available bandwidth provided by each access or more efficient sort [14].

Considering the contexts in mobility, where the QoS is subjected to high variability, it has been necessary to combine this technique with an adaptive load balancing mechanism able to vary dynamically the load in a manner proportional to the estimated available bandwidth on each data access.

To measure the available bandwidth, estimation tools based on non-intrusive technique such as Self Loading of Periodic 


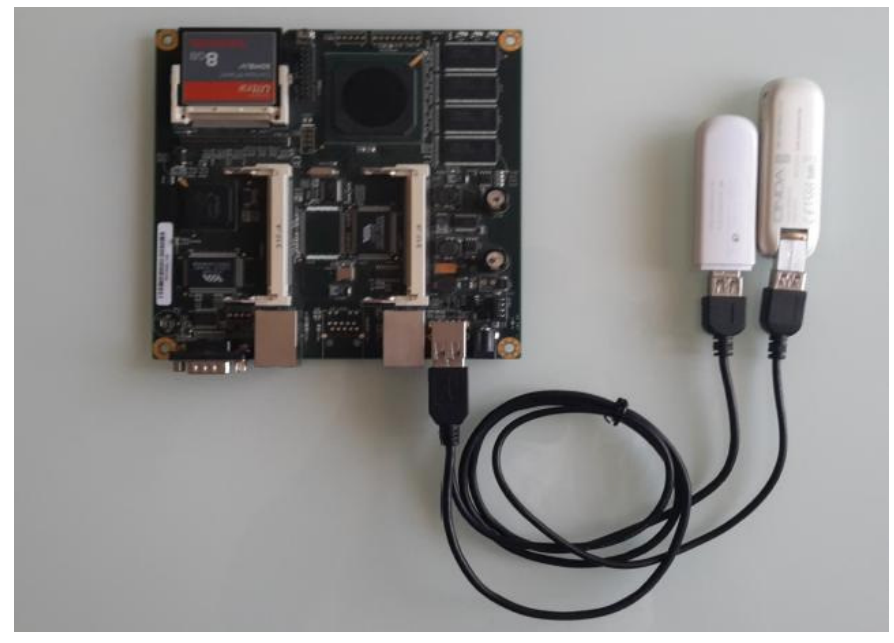

Fig. 2. Prototype employed during test campaign [2]

Stream (SLoPS) techniques have been adopted [15], [16], [17].

Thanks to the adoption of these techniques a fairly accurate estimate of the available bandwidth was made, but unfortunately these techniques have relatively long convergence time, which could prove to be inadequate in high mobility contexts where the access conditions may change rapidly.

In the following subsection the performance, advantages and issues of Smart VPN Bonding technique are presented.

\section{B. Performance evaluation}

In order to evaluate the performance of Smart VPN bonding technique we report the results of a test campaign [1], [2].

The tests were carried out using a prototype based on ALIX2D2 board, a system board optimized for routing and network applications; two USB Internet Keys equipped with two SIMs of different mobile network operators (called Operator 1 and Operator 2) to provide cellular connectivity; ZeroShell and OpenVPN has been used as operative systems and VPN manager respectively; finally a proprietary script has been realized by using bash and python language to evaluate the end-to-end available bandwidth and, consequently, to establish the weights to assign to load balancing mechanism. The experimental prototype is shown in Fig. 2, while the Smart VPN Bonding behavior is depicted in Fig. 3.

The performances are obtained in terms of throughput measured using some FTP sessions over the two Internet accesses in 15 different geographic test points. In this scenario the QoS offered by Operator 1 is comparable to Operator 2. The Fig. 4 shown the average values obtained. In this case both operators provide a high QoS, so a static approach based on round robin strategy applied to load balancing mechanism represents a good solution, increasing performance by more than $50 \%$ if compared to the best mobile operator.

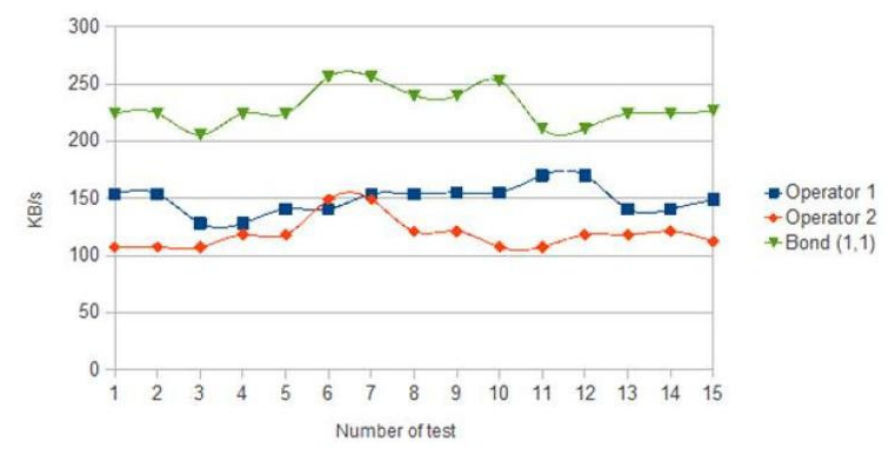

Fig. 3. Throughput measured in good network conditions for the Operator 1, Operator 2 and bonding interface

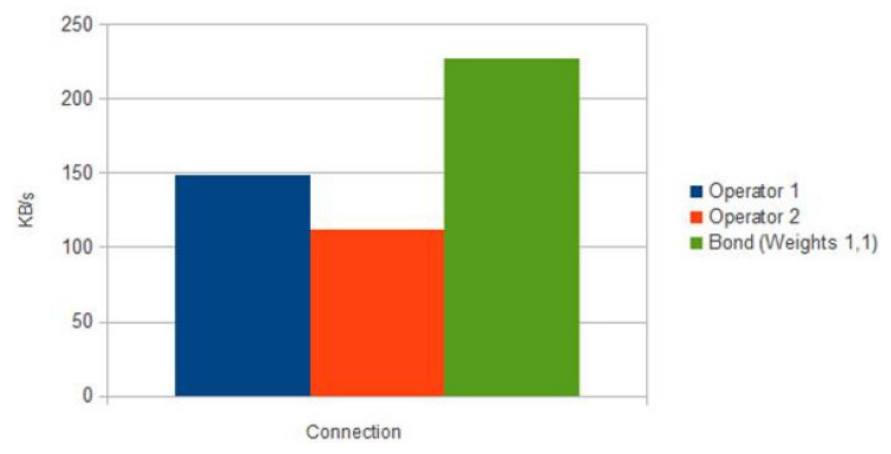

Fig. 4. Average throughput comparison

The performance delivered by the VPN bonding coupled with the static load balancing between the two available Internet accesses is satisfactory in the above mentioned scenarios. However, this technique has some disadvantages when the bandwidth offered by two operators is not similar.

Fig. 5 shows a particular case in which the available bandwidth provided by Operator 1 is affected by a considerable degradation due to a poor radio coverage. In this case a static weights assignment to load balancing mechanism don't represent the best approach (Fig. 5a). Indeed, the bonding interface behavior is similar to the worst mobile operator, so the VPN bonding technique does not offer any performance improvement because of the incorrect weights assignment. Instead, using an adaptive weights assignment in order to counteract the drawbacks related to the variability of the end-to-end bandwidth offered by each radio operator along the path the performance in terms of throughput is considerably enhanced (Fig. 5b). The scenarios above mentioned highlight the advantages and bandwidth improvement provided by Smart VPN Bonding. However, a limit of this technique is represented by high response time to react rapidly to changing network conditions due to bandwidth estimation tool. Fig. 6 shows the performance of Smart VPN Bonding technique transmitting a large file during a change of location site, from a good to poor radio coverage areas for one of two mobile operators. 


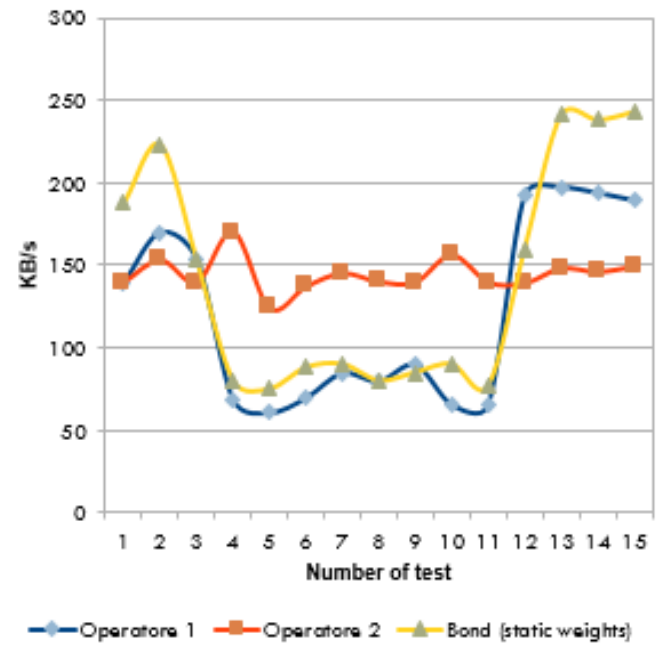

(a)

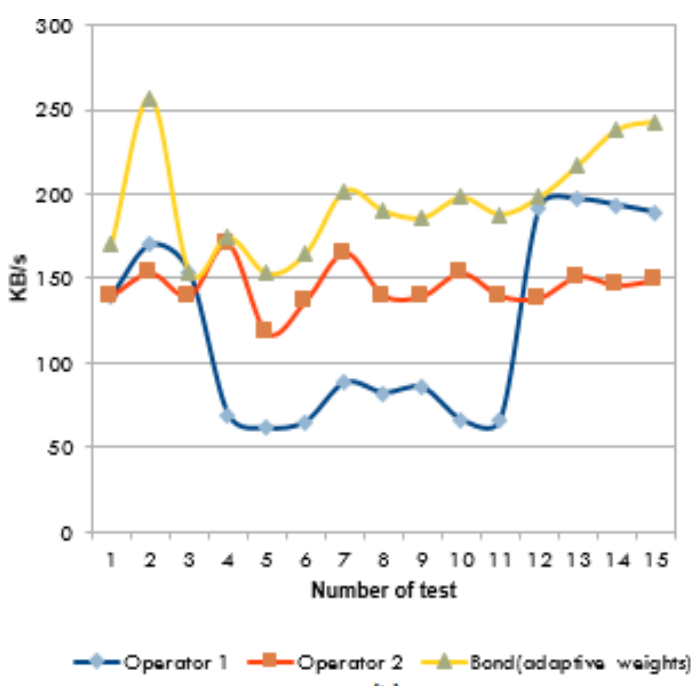

(b)

Fig. 5. Operator 1 bandwidth degradation: (a) static weights, (b) adaptive weights

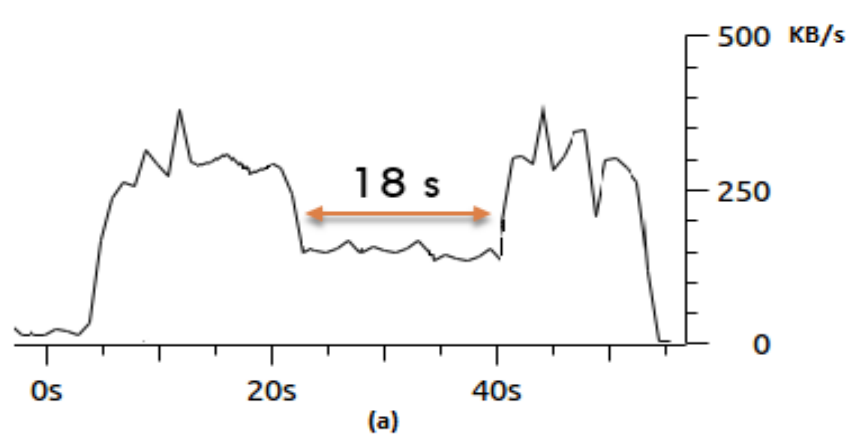

Fig. 6. Smart VPN Bonding time response

After the change of location site the throughput measured on bonding interface is considerably lower caused by incorrect load balancing. From the example illustrative in Fig. 6, the system has required about 18 seconds to estimate the available bandwidth offered by both internet accesses, to recognize the changing network conditions and modify the weights assignment to load balancing mechanism. In fact, as soon as the change of weights has occurred, the throughput on bonding interface increases.

This high response time has encouraged the authors to investigate the relation between RTT and available bandwidth in a specific geographic location, in order to obtain an accurate bandwidth estimation in a very short time. Indeed, the RTT measurement is very easy and much faster than available bandwidth estimation, and even more suitable in high mobility context.

In order to verify and analyze this relation a Neural Network approach was adopted.

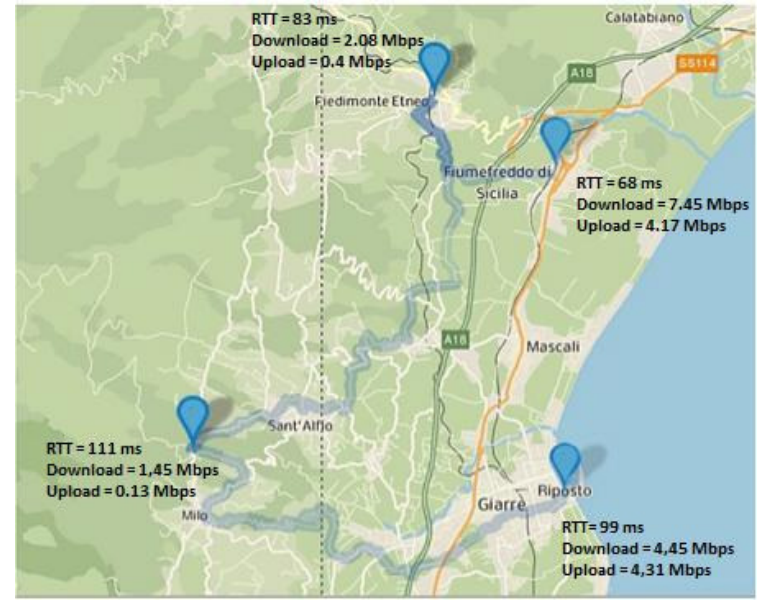

Fig. 7. Testbed Scenario

The dataset for the Neural Network training includes values of RTT, download end-to-end available bandwidth and upload end-to-end available bandwidth calculated every 5 minutes. To filter out any episodic RTT effects, each RTT measurement was calculated as the median value of 5 individual samples spaced 2 seconds apart. The available endto-end bandwidth measurements were carried out using Pathchirp tool.

\section{DATASET}

The dataset for Neural Network training includes the data collected in an urban scenario, as shown in Fig. 7. Along the path shown in Fig. 7, the test points have been selected to 


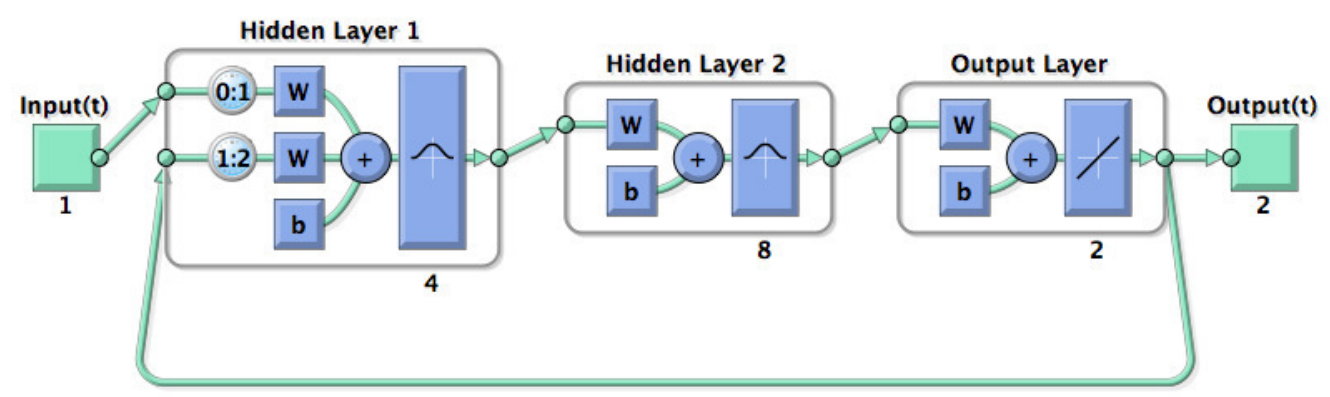

Fig. 8. The RNN used for the prediction of the upload and download rates.

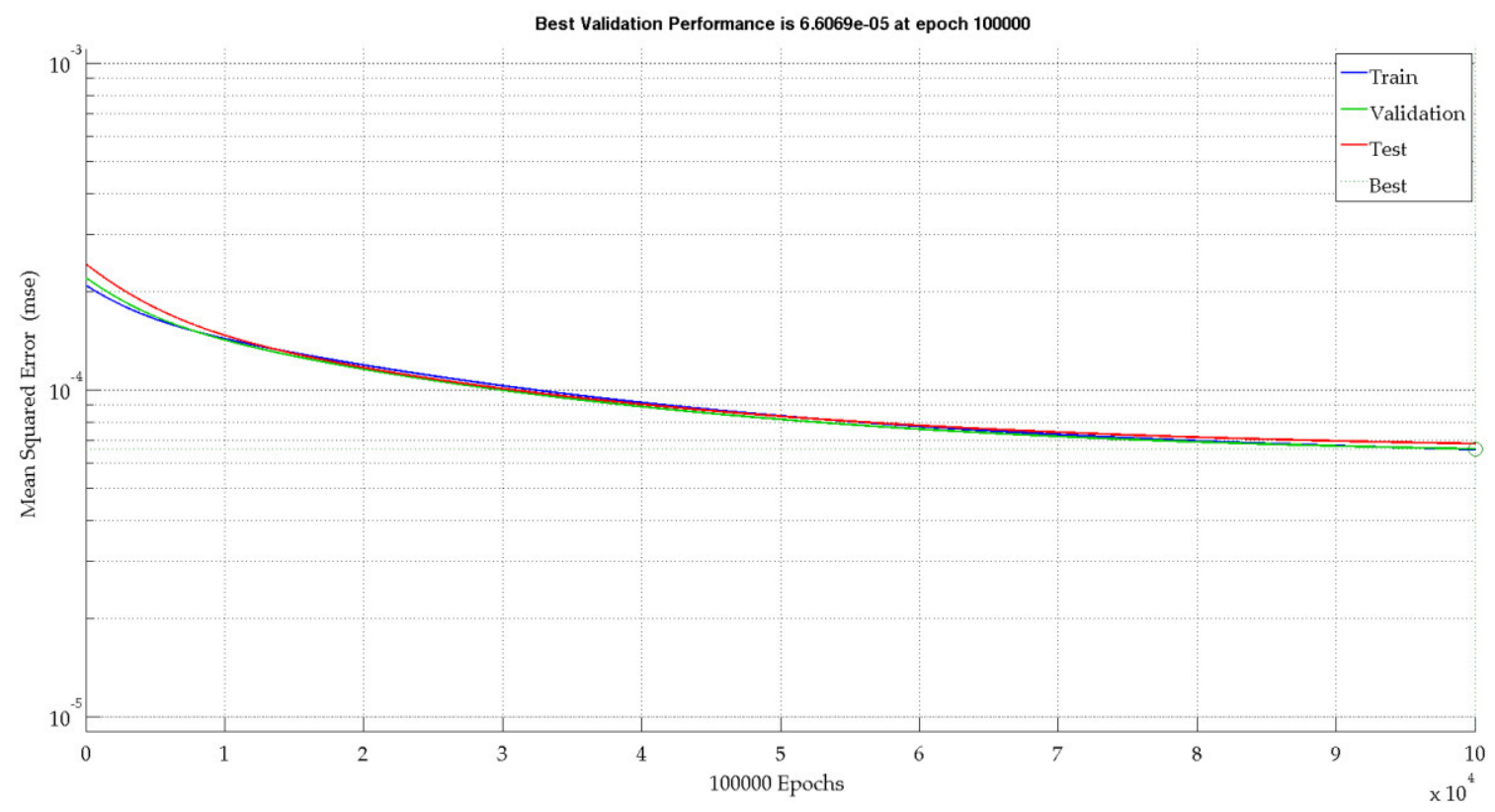

Fig. 9. Learning curves of the Recurrent Neural Network.

measure RTT, end-to-end upload and download bandwidth. In Fig. 7 are reported just some examples of measuring points.

As mentioned in Section III, the end-to-end available bandwidth estimation tools have long convergence time, inadequate for high mobility contexts, so the relationship between available bandwidth values and RTT is analyzed, in order to adopt RTT measurement to predict the available bandwidth values. For each test point, 60 values of RTT, endto-end upload and download available bandwidth have been collected for the training of the Neural Network. In Section V the results and the performance for one test point are reported. These results can be extended to other test points thanks to the generalization ability of the Neural Network.

\section{NEURAL NETWORK PERFORMANCE}

Different topologies of Neural Network were experimented in order to gain some insight into the most appropriate network architecture [18], [19], [20], [21].

The best conducted experiments in terms of MSE, nooverfitting and generalization were obtained using a real-time Recurrent Neural Network (RNN) as depicted in Fig. 8. The RNN is composed by an input layer, an output layer and two hidden layers: the four neurons of the first hidden layer and the eight neurons of the second hidden layer have radial basis transfer function. While for the two neurons of the output layer has been used a linear transfer function. The RTT time series is used as input vector while the upload and download rate time series are used as output vectors. The input vector is delayed with zero step delay and one step delay while the output vectors are delayed with one step delay and two step delay. So the RNN predicts the values of upload and download rates at time $t_{0}+1$ based on the value of the RTT at time $t_{0}$ and 
$t_{0}-1$. The time step in this paper is one minute. The learning curves, shown in Fig. 9, pointed out the good performance of the RNN reached after 100000 epochs with a mean squared error of 6.6e- 05 and an excellent generalization due to the fact that the test curve is always very close to the training curve.

\section{CONCLUSIONS}

In this paper we have investigated the relation between the Round Trip Time (RTT) and the end-to-end available bandwidth (upload and download) in order to simplify and speed up the estimation bandwidth process.

The results highlight that it is possible to estimate the available bandwidth based on the knowledge of the past values of the RTT obtaining a low MSE. Thanks to this information it is possible to apply a fast reconfiguration of weights of the load balancing mechanism adopted in VPN bonding technique to guarantee a higher end-to-end available bandwidth than a static approach (e.g. round robin strategy). This approach is very useful to improve the VPN bonding performance, but can be used in several other application scenarios for the important adaptation of available bandwidth (e.g. video transmission frame rate).

\section{REFERENCES}

[1] Beritelli, F., La Corte, A., Rametta, C., Scaglione, F. (2015). A Cellular bonding and adaptive load balancing based multi-sim gateway for mobile ad hoc and sensor networks. International Journal on Ad Hoc Networking Systems (IJANS), 5(3).

[2] Beritelli, F., La Corte, A., Lo Sciuto, G., Rametta, C., Scaglione, F. (2016). Adaptive VPN Bonding Technique for Enhancing Dual-SIM Mobile Internet Access. In: Proceedings of the International Symposium for Young Scientists in Technology, Engineering and Mathematics - SYSTEM - Catania, Italy, September 27-29, 2015. p. 47-54.

[3] Beritelli F, Rametta C, Raspanti A, Russo M, Scaglione F, Spallina G (2016). An advanced QOS analysis and evaluation method for mobile internet access. International Journal of Wireless and Mobile Networks, 2016, vol. 8, p. 55-70.

[4] Liu, Liangwen, and Jipeng Zhou. "Ad hoc on-demand QoS routing based on bandwidth prediction (AQBP)." $20128^{\text {th }}$ IEEE International Conference onWireless Communications, Networking and Mobile Computing (WiCOM), 2012.

[5] Salih, Yass K., Ong Hang See, and Salman Yussof. "A fuzzy predictive handover mechanism based on MIH links triggering in heterogeneous wireless networks." International Conference on Software and Computer Applications (ICSCA). Vol. 41. 2012.

[6] Miyim, A. M., Ismail, M., Nordin, R., Mahardhika, G. "Generic vertical handover prediction algorithm for $4 \mathrm{G}$ wireless networks". In Space Science and Communication (IconSpace), 2013 IEEE International Conference on (pp. 307-312).

[7] Reddy, K. Suresh Kumar, D. Rajaveerappa, and S. KhadeejaBanu. "Bandwidth Map-TCP friendly rate control algorithm for improving QoS in streaming applications." 2013 Fourth International Conference on. Computing, Communications and Networking Technologies (ICCCNT), 2013.

[8] Yao, Jun, Salil S. Kanhere, and Mahbub Hassan. "Improving QoS in high-speed mobility using bandwidth maps." IEEE Transactions on Mobile Computing 11.4 (2012): 603-617.
[9] Landa, Raul, et al. "Measuring the relationships between internet geography and rtt." Computer Communications and Networks (ICCCN), 2013 22nd International Conference on. IEEE, 2013.

[10] Iliya, S., Goodyer, E., Gongora, M., Shell, J., \& Gow, J. "Optimized artificial neural network using differential evolution for prediction of RF power in VHF/UHF TV and GSM 900 bands for cognitive radio networks." Computational Intelligence (UKCI), 2014 14th UK Workshop on. IEEE, 2014.

[11] Iliya, S., Goodyer, E., Gow, J., Shell, J., \& Gongora, M. "Application of Artificial Neural Network and Support Vector Regression in cognitive radio networks for RF power prediction using compact differential evolution algorithm." Computer Science and Information Systems (FedCSIS), 2015 Federated Conference on. IEEE, 2015.

[12] Chaudhari, Shilpa Shashikant, and Rajashekhar C. Biradar. "Available bandwidth prediction using wavelet neural network in mobile ad-hoc networks." Circuits, Communication, Control and Computing (I4C), 2014 International Conference on. IEEE, 2014.

[13] Chaudhari, Shilpa Shashikant, and Rajashekhar C. Biradar, "Resource prediction using wavelet neural network in mobile adhoc networks." Advances in Electronics, Computers and Communications (ICAECC), 2014 International Conference on. IEEE, 2014.

[14] Marszalek, Z., "Novel Recursive Fast Sort Algorithm" Information and Software Technologies - 22nd International Conference, ICIST 2016, Druskininkai, Lithuania, October 1315, 2016, Proceedings, 2016, pp. 344-355.

[15] Strauss, J., Katabi, D., and Kaashoek, F., "A measurement study of available bandwidth estimation tools." Proceedings of the 3rd ACM SIGCOMM conference on Internet measurement. ACM, 2003.

[16] Ribeiro, V. J., Riedi, R. H., Baraniuk, R. G., Navratil, J., Cottrell, L. "Pathchirp: Efficient available bandwidth estimation for network paths." Passive and active measurement workshop. 2003.

[17] Manish, J. and Dovrolis, C. "Pathload: A measurement tool for end-to-end available bandwidth." In Proceedings of Passive and Active Measurements (PAM) Workshop. 2002.

[18] Capizzi, G., Lo Sciuto, G., Napoli, C., Tramontana, E. "A multithread nested neural network architecture to model surface plasmon polaritons propagation.” 2016 Micromachines, 7 (7), art. no. 110.

[19] Bonanno, F., Capizzi, G., Lo Sciuto, G., "A neuro wavelet-based approach for short-term load forecasting in integrated generation systems."2013 4th International Conference on Clean Electrical Power: Renewable Energy Resources Impact, ICCEP 2013, pp. 772-776.

[20] G. Capizzi, G. Lo Sciuto, C. Napoli, E. Tramontana and M. Woźniak, "Automatic classification of fruit defects based on cooccurrence matrix and neural networks," 2015 Federated Conference on Computer Science and Information Systems (FedCSIS), Lodz, 2015, pp. 861-867.

[21] Capizzi, G., Lo Sciuto, G., Woźniak, M. and R. Damaševicius, "A Clustering Based System for Automated Oil Spill Detection by Satellite Remote Sensing," 2016 International Conference on Artificial Intelligence and Soft Computing, ICAISC 2016: Artificial Intelligence and Soft Computing pp 613-623.

[22] Sroczyński, Z., "Actiontracking for Multi-platform Mobile Applications," Software Engineering Trends and Techniques in Intelligent Systems, CSOC 2017, pp. 339-348. 